\title{
ON HERMITIAN STRUCTURES OF PRESCRIBED NONPOSITIVE HERMITIAN SCALAR CURVATURE
}

\author{
BY MELVYN S. BERGER
}

Communicated by Philip Hartman, January 18, 1972

1. Introduction. Let $(M, g)$ be a given Kähler metric on a compact manifold $M$ of complex dimension $N$. If one denotes the associated volume element by $d V_{q}$ and the scalar curvature by $k_{g}$, then it is known [1, p. 118] that $c(M)=\int_{M} k_{g} d V_{g}$ is a Kähler invariant (i.e. is independent under Kähler deformation of the particular Kähler metric $g$ defined on $M$ ). Here we give some results on the following problem:

$\left(\pi_{N}\right)$ Find necessary and sufficient conditions on a Hölder continuous function $K(x)$ defined on $(M, g)$ such that $K(x)$ is the Hermitian scalar curvature of some Hermitian metric $\bar{g}$ on $M$ conformally equivalent to $\bar{g}$.

If $N=1$, this problem was studied by the author in [2], and subsequently in [3] by Kazdan and Warner. However the methods used in these papers depend crucially on the fact that $N=1$. Indeed, certain calculus inequalities for the functions $u$ in the Sobolev space $W_{1,2}(M, g)$ are required, that hold in case $N=1$ but not otherwise.

2. The main result. We seek a smooth real-valued function $u(x)$ defined on $(M, g)$ such that the Hermitian scalar curvature $k_{\tilde{g}}(x)$ of $M$ with respect to the Hermitian metric $\tilde{g}=e^{2 u} g$ is the given function $K(x)$. By means of the results of Chern [4], and Chavel [5], the function $u(x)$ is a solution of the semilinear elliptic equation

$$
N \Delta u-k_{g}(x)+K(x) e^{2 u}=0
$$

where $\Delta$ is the Laplace-Beltrami operator relative to $(M, g)$. By integrating (1) over $M$, we find that a necessary condition for the solvability of (1) is that

$$
c(M) \equiv \int k_{g}(x) d V_{g}=\int K(x) e^{2 u} d V_{g} .
$$

By the remarks of the Introduction, this relation is invariant under Kähler deformation of $g$, and is an analogue of the Gauss-Bonnet formula for $N=1$. As in [2], (2) may be used to formulate isoperimetric variational problems whose solutions (if they exist) satisfy (1). However, if $N>1$, the solvability of these isoperimetric problems is in question, so an

AMS 1970 subject classifications. Primary 35J20, 53A30.

Copyright (C) American Mathematical Society 1972 
alternative approach is required. Nonetheless one can prove the following results for the problem $\left(\pi_{N}\right)$ :

THEOREM 1. Suppose $c(M)<0$, then a sufficient condition for the solvability of (1) is that the Hölder continuous function $K(x)$ be nonpositive but not identically zero. A necessary condition for the solvability of (1) is that $\int K(x) e^{2 w} d V_{g}<0$ where $w$ satisfies

$$
N \Delta w=k_{g}(x)-[\operatorname{vol}(M, g)]^{-1} c(M) .
$$

Corollary 2. Suppose $c(M)<0$, then any Hölder continuous nonpositive and nonidentically vanishing function is the Hermitian scalar curvature for a Hermitian metric $\bar{g}$ conformal to $g$.

IDEA OF PROOF OF THEOREM 1. The proof proceeds by first assuming $k_{g}(x)$ equals the constant $k_{g} \equiv[\operatorname{vol}(M, g)]^{-1} \int k_{g}(x) d V_{g}$ and $\int-K(x) d V_{g}$ is sufficiently large. Then the functional

$$
I(u)=\int_{M}\left\{\frac{1}{2} N|\nabla u|^{2}+k_{g}(x) u-\frac{1}{2} K(x) e^{2 u}\right\} d V_{g}
$$

achieves its infimum over the admissible class $C=W_{1,2}(M, g)$ (i.e. the set of all square-integrable functions over $(M, g)$ which also possess a square integrable gradient, $\nabla u$ ), at the element $u_{0} \in W_{1,2}(M, g)$ (say). It is then shown that the element $u_{0}$ is smooth and satisfies (1) by showing that ess $\sup \left|u_{0}\right|$ is bounded over $M$ and applying the regularity theory for linear elliptic equations. The general case is then reduced to this special case above by writing a tentative solution of (1) $w=u+v$ where $u$ and $v$ satisfy the system:

$$
\begin{gathered}
N \Delta u-\bar{k}_{g}+K e^{2 v} e^{2 u}=0, \\
N \Delta v-k_{g}(x)+\bar{k}_{g}=0 .
\end{gathered}
$$

Clearly, since $v$ is uniquely determined only up to an additive constant by (4), equation (3) satisfies the hypotheses of the special case just discussed.

The necessary condition of the theorem is obtained as in [3], by multiplying the equation (1) by $e^{-2 u}$, integrating over $M$ and integrating by parts, in case $k_{g}(x)=$ const. In the general case, we repeat this argument for equation (3).

REMARK If $K(x) \equiv 0$, then it is standard to show that (1) is solvable if and only if $\int k_{g}(x) d V_{g}=0$.

ADDED IN PROOF. Recent results of Kazdan and Warner indicate that the conclusion of Corollary 2 may be extended to read: any Hölder continuous function, negative at some point of $M$ is the Hermitian scalar curvature for a Hermitian metric conformal to $g$ provided conformal 
equivalence is understood in a sense more general than the pointwise notion used here.

\section{BIBLIOGRAPHY}

1. Marcel Berger, P. Gauduchon and E. Mazet, Le spectre d'une variété Riemannienne, Lecture Notes in Math., vol. 194, Springer-Verlag, New York, 1970.

2. M. S. Berger, Riemannian structures of prescribed Gaussian curvature for 2-manifo'ds, J. Differential Geometry 5 (1971), 325-332.

3. J. Kazdan and F. Warner, Curvature functions for 2-manifolds. I (to appear).

4. S. S. Chern, On holomorphic mappings of Hermitian manifolds of the same dimension, Proc. Sympos. Pure Math. vol. 11, Amer. Math. Soc., Providence, R.I., 1968, pp. 157-170. MR 38 \# 2714.

5. I. Chavel, Two variational problems in Hermitian geometry, Indiana Univ. Math. J. 20 (1970/71), 175-183. MR 41 \#6127.

Belfer Graduate School of Science, Yeshiva University, New York, New York 10033

Current address: School of Mathematics, Institute for Advanced Study, Princeton, New Jersey 08540 